\title{
Recht auf Akteneinsicht
}

Eines der zentralen Patientenrechte ist das Recht auf Akteneinsicht. Das Recht des Patienten auf Selbstbestimmung und dessen personale Würde gebieten es, dass dieser gegenüber Arzt und Krankenhaus einen Anspruch auf Einsicht in die ihn betreffenden Krankenunterlagen hat, ohne dass er hierfür ein besonderes rechtliches Interesse darlegen müsste. Auf Seiten des Arztes korrespondiert mit diesem Recht auf Akteneinsicht die ärztliche Pflicht zur Gewährung von Akteneinsicht - sowohl aus Behandlungsvertrag als auch berufsrechtlich (vgl. $\$ 10$ Abs. 2 MBO- ̈ - Musterberufungsordnung Ärzte).

Dem Patientenrecht auf Akteneinsicht entspricht im allgemeinen Datenschutzrecht das Recht des von der Datenverarbeitung Betroffenen auf Auskunft über die zu seiner Person gespeicherten Daten (\$\$ 19, 34 BDSG). Dieses Auskunftsrecht wird ganz allgemein als das fundamentale Datenschutzrecht für den Betroffenen eingeordnet; es versetzt den Einzelnen überhaupt erst in die Lage, sein informationelles Selbstbestimmungsrecht auch im Sinne eines Kontrollrechts einzusetzen und ggf. auf eine Löschung, Berichtigung oder Sperrung seiner Daten hinzuwirken. Gemäß $₫ 6$ Abs. 1 BDSG ist das Auskunftsrecht unabdingbar und kann nicht durch Rechtsgeschäft ausgeschlossen oder beschränkt werden.

\section{Inhalt}

Das Recht auf Akteneinsicht verpflichtet den Arzt nicht zur Herausgabe der Originalunterlagen, sondern lediglich zur Einsichtgewährung. Grundsätzlich ist es die Entscheidung des Arztes, auf welche Art und Weise er dem Patienten Einsicht in die Unterlagen gewähren möchte. Er kann seiner Verpflichtung insbesondere auch dadurch nachkommen, dass er dem Patienten entsprechende Kopien der Patientenunterlagen herausgibt - Zug um Zug gegen Erstattung der hierfür anfallenden Kosten.

\section{Einschränkungen}

Ausnahmsweise kann das Recht des Patienten auf Akteneinsicht eingeschränkt werden, wenn sich bei Kenntnis des Patienten vom Akteninhalt negative gesundheitliche Konsequenzen für den $\mathrm{Pa}$ tienten ergeben können (sog. therapeutischer Vorbehalt). Insbesondere in den Bereichen Psychiatrie und Psychotherapie kann eine Einsicht in Krankenaufzeichnungen und Gesprächsprotokolle unter Umständen den Therapieerfolg gefährden oder die Befindlichkeit des Patienten negativ beeinflussen.

Auch wenn sich Arzt und Krankenhaus auf einen therapeutischen Vorbehalt stützen können, heißt dies aber nicht, dass sie überhaupt keine Einsicht in die Behandlungsunterlagen gewähren müssen. Vielmehr kann den therapeutischen Bedenken des Arztes im Einzelfall auch dadurch Rechnung getragen werden, dass die Einsichtnahme nicht dem Patienten selbst gestattet wird, sondern die Unterlagen an einen anderen Arzt ausgehändigt werden, der dann in eigener Verantwortung entscheidet, welche Informationen auch dem Patienten selbst zugänglich gemacht werden können.

Nach $\$ 10$ Abs. 2 MBO-Ä sind vom Patientenrecht auf Akteneinsicht auch diejenigen Teile der Krankenunterlagen ausgenommen, welche „subjektive Eindrücke oder Wahrnehmungen der Ärztin oder des Arztes enthalten“. Auch diese Einschränkung ist vor allem in den Bereichen Psychiatrie und Psychotherapie von Bedeutung, wenn es um die Aufzeichnungen des Behandelnden über seine subjektiven Wahrnehmungen geht, in denen er zum Zweck der Selbstkontrolle seine eigenen Gefühle und Gedankengänge niedergelegt hat (sog. „Gegenübertragungen“).

Wiederum gilt, dass aber nach Möglichkeit das Einsichtsrecht des Patienten nicht völlig ausgeschlossen werden soll. $\mathrm{Zu}$ prüfen ist vielmehr, inwieweit dem Persönlichkeitsschutz des Behandelnden dadurch Rechnung getragen werden kann, dass bestimmte Aktenteile herausgenommen, geschwärzt oder bei der Kopie abgedeckt werden.

\section{Rechte Dritter auf Einsichtnahme}

Anerkannt ist, dass das Recht auf Einsichtnahme in bestimmten Konstellationen auch auf Dritte übertragen werden kann. Stets ist jedoch die Schweigepflicht des Arztes als Schranke zu beachten. Nur wenn eine Einwilligung des Betroffenen in die Einsichtnahme durch Dritte feststeht oder ausnahmsweise eine mutmaßliche Einwilligung des Betroffenen angenommen werden kann, ist der Arzt insoweit von seiner Schweigepflicht entbunden.

Typisches Beispiel ist der Auskunftsanspruch einer Krankenkasse: Wenn eine Krankenkasse wegen einer Körperschädigung dem Geschädigten Sozialleistungen gewähren muss, gehen kraft Gesetzes die Schadensersatzansprüche des Geschädigten auf die Krankenkasse über (siehe $\$ 116$ Abs. 1 SGB X). Damit verbunden ist dann auch ein Übergang des Akteneinsichtsrechts als sog. "Hilfsrecht“. Voraussetzung ist jedoch, dass die ärztliche Schweigepflicht beachtet wird. Stets muss daher eine Einwilligung des betroffenen Patienten in eine Datenweitergabe zu bejahen sein - entweder eine ausdrückliche oder ausnahmsweise eine mutmaßliche, soweit einer ausdrücklichen Einwilligung Hindernisse entgegenstehen.

Die Schweigepflicht des Arztes gilt auch gegenüber nahen Angehörigen und zwar über den Tod des Patienten hinaus. Ein Einsichtsrecht steht nahen Angehörigen daher nur ausnahmsweise und lediglich im vermuteten Einverständnis des Patienten $\mathrm{zu}$, soweit der Patient nicht eine ausdrückliche Einwilligung erteilen konnte. Es ist dann Aufgabe des Arztes, sich die Gewissheit zu verschaffen, ob der Patient vor seinen Angehörigen keine Geheimnisse über seinen Gesundheitszustand haben will bzw. hätte haben wollen.

Gegenüber den Eltern eines minderjährigen Patienten ist der Arzt dann an seine Schweigepflicht gebunden, wenn der Minderjährige selbst bereits eine entsprechende Einsichtsfähigkeit besitzt. Ab welchem Alter eine solche Einsichtsfähigkeit anzunehmen ist, hängt vom konkreten Einzelfall ab. 